\title{
Hepatocellular Carcinoma Metastasis to the Foot
}

\author{
(1) Deniz SEYITHANOĞLU,' (1) Bilger ÇAVUŞ, ${ }^{2}$ (D) Aslı ÇiFÇiBAŞı ÖRMECi, ${ }^{2}$ (D) Nesimi BÜYÜKBABANi, ${ }^{3}$ \\ (D) Arzu POYANLI, ${ }^{4}$ (D) Sabahattin KAYMAKOĞLU²
}

\begin{abstract}
'Department of Internal Medicine, Istanbul University Faculty of Medicine, Istanbul-Turkey
${ }^{2}$ Department of Gastroenterohepatology, Istanbul University Faculty of Medicine, Istanbul-Turkey

${ }^{3}$ Department of Pathology, Istanbul University Faculty of Medicine, Istanbul-Turkey

${ }^{4}$ Department of Radiology, Istanbul University Faculty of Medicine, Istanbul-Turkey
\end{abstract}

\section{Dear Editor,}

Hepatocellular carcinoma (HCC) is the most common primary liver cancer.[1] The most common extrahepatic sites for HCC are the lungs, abdominal lymph nodes, and bones.[1] Acrometastases are rare among HCC metastases. This letter describes a patient with HCC metastasis to the big toe.

A 67-year-old man with chronic hepatitis B infection without an ongoing treatment presented with a soft lesion on the left big toe which had grown as large as a walnut, with a blue-purple discoloration. His recent alpha-fetoprotein was 2.1 , liver elastography with FibroScan revealed F3 fibrosis stage, aspartate transaminase to platelet ratio index was 0.3 , and liver function tests were normal. The lesion was already excised at another center for bleeding after trauma. Re-examination of the material revealed tumoral structures with bile pigments between dilated vascular structures in the dermis (Fig. 1). Immunohistochemistric staining revealed metastatic HCC with micro-trabecular and pseudoacinar pattern; staining positive with Hep Par-1, CD 10, Glutamine Synthetase, Cytokeratin, and Glypican-3. Magnetic resonance imaging (MRI) revealed a T1A hipointense, T2A hyperintense $27 \mathrm{~mm} \times 23 \mathrm{~mm}$ lesion that had destroyed the first distal phalanx (Fig. 2). An upper abdomen MRI with IV gadolinium-based contrast showed a $25 \mathrm{~mm} \times 22 \mathrm{~mm}$ lesion at the $5^{\text {th }}$ segment of the liver. The patient was discussed at a multidisciplinary council; the liver lesion was deemed as HCC; and transarterial chemoembolization was done. The left toe was amputated from the proximal phalanx by orthopedic surgeons. Sorafenib treatment was commenced by oncology. The patient is currently followed in the outpatient setting and has given consent for the publication of this letter.

Acrometastases are seen in $0.1 \%$ of bone metastases; almost half are due to pulmonary cancer.[2] Tumor microemboli through arterial circulation, rather than lymphatic dissemination, are thought to explain acrometastasis in pulmonary cancer.[3] Nevertheless, systemic embolization is harder for visceral tumors like HCC that need to pass through the pulmonary capil-

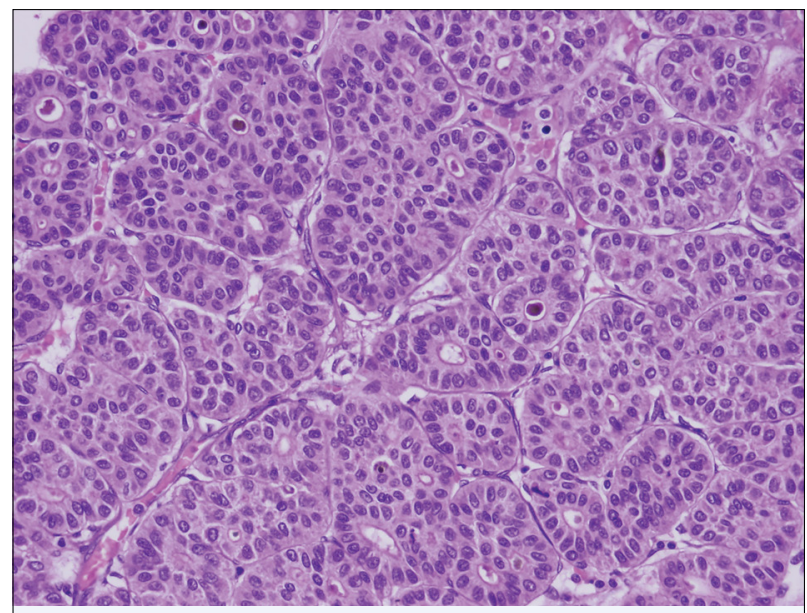

Fig. 1. Tumor with pseudo acinar and trabecular structures in deeper dermis with dilated vascular structures and bile plugs inside dilated pseudo acinar structures (hematoxylin and eosin staining, magnification, $\mathrm{x} 400$ ).
Dr. Deniz SEYITHANOĞLU

İstanbul Üniversitesi Tıp Fakültesi,

Iç Hastalıkları Anabilim Dalı,

Istanbul-Turkey

E-mail: deniz.seyithanoglu@istanbul.edu.tr 


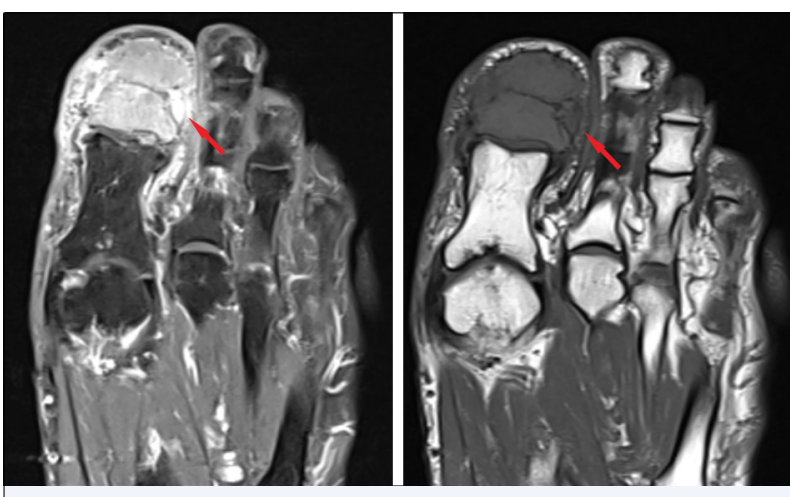

Fig. 2. MRI of the left foot showing the lesion at the first distal phalanx; cross-sectional view T1A and T2A.

lary system.[3] Distal phalanx is commonly affected, which could be due to slowing of the blood circulation at capillaries, facilitating microemboli settling.[4] Releasing of chemotactic factors following trauma could also draw tumor cells to the site and increase adherence; which could explain the acrometastasis in our patient.[4]

Acrometastases present as rapidly growing palpable masses causing intermittent pain and mechanical disfunction.[3] HCC's are hypervascular tumors which are prone to bleeding spontaneously or during biopsy.[5] MRI is the best method for evaluating HCC acrometastases, which are differentiated by their osteolytic characteristics.[4] Acrometastases are usually found with widespread disease with poorer outcome. [3] In advanced patients; treatment is mostly palliative; aimed at controlling pain and regaining function.[5]
For lesions limited to the distal phalanx; amputation is frequently used.[4] The use of systemic chemotherapy is debatable but has been used in some cases.[2,4] Amputation and chemotherapy were chosen for our patient due to his normal performance status.

\section{Conclusion}

HCC may present as painful, palpable mass on the distal phalanx in chronic hepatitis B patients. Concurrent liver and full-body imaging are necessary to find the primary tumor and other metastatic sites. Suitable treatments should be tailored for each case according to localization of the lesion, performance status and liver function tests.

\section{References}

1. Katyal S, Oliver JH $3^{\text {rd }}$, Peterson MS, Ferris JV, Carr BS, Baron RL. Extrahepatic metastases of hepatocellular carcinoma. Radiology 2000;216(3):698-703.

2. Rauf MS, Motta L, Connolly C. Digital acrometastases as first sign of hepatocellular carcinoma. Scott Med J 2012;57(4):247.

3. Flynn CJ, Danjoux C, Wong J, Christakis M, Rubenstein J, Yee A, et al. Two cases of acrometastasis to the hands and review of the literature. Curr Oncol 2008;15(5):51-8.

4. Stomeo D, Tulli A, Ziranu A, Perisano C, De Santis V, Maccauro G. Acrometastasis: a literature review. Eur Rev Med Pharmacol Sci 2015;19(15):2906-15.

5. Lee KS, Lee SH, Kang KH, Oh KJ. Metastatic hepatocellular carcinoma of the distal phalanx of the thumb. Hand Surg 1999;4(1):95-100. 